\title{
Dominique BERTHET, Les défis de la critique d'art
}

Paris, Éd. Kimé, coll. Esthétiques, 2006, 122 p.

Jean-Marc Lachaud

\section{OpenEdition}

\section{Journals}

\section{Édition électronique}

URL : http://journals.openedition.org/questionsdecommunication/7745

DOI : $10.4000 /$ questionsdecommunication. 7745

ISSN : 2259-8901

\section{Éditeur}

Presses universitaires de Lorraine

\section{Édition imprimée}

Date de publication : 1 décembre 2006

ISBN : 978-2-86480-828-2

ISSN : 1633-5961

\section{Référence électronique}

Jean-Marc Lachaud, "Dominique BeRTHEт, Les défis de la critique d'art », Questions de communication [En ligne], 10 | 2006, mis en ligne le 01 décembre 2006, consulté le 22 mars 2021. URL : http:// journals.openedition.org/questionsdecommunication/7745 ; DOI : https://doi.org/10.4000/ questionsdecommunication.7745

Ce document a été généré automatiquement le 22 mars 2021.

Tous droits réservés 


\section{Dominique BERTHET, Les défis de la critique d'art}

Paris, Éd. Kimé, coll. Esthétiques, 2006, 122 p.

Jean-Marc Lachaud

\section{RÉFÉRENCE}

Dominique BERTHET, Les défis de la critique d'art. Paris, Éd. Kimé, coll. Esthétiques, 2006, $122 \mathrm{p}$.

Dominique Berthet, philosophe, esthéticien, enseignant-chercheur et directeur de l'incontournable revue Recherches en esthétique, développe, dans cet ouvrage qui cerne et discute Les défis de la critique d'art, une rigoureuse approche de nombreuses hypothèses, anciennes ou actuelles, s'attachant à définir l'art de la critique et à décrypter les enjeux que celle-ci, inéluctablement, soulève et auxquels elle doit répondre. Incontestablement, ce livre se nourrit d'une riche expérience personnelle dans le domaine de la critique, puisque Dominique Berthet est également critique d'art! Par exemple, il vient de publier une très forte étude sur l'originale démarche d'un artiste martiniquais, Ernest Breleur, qui a abandonné la peinture pour utiliser la radiographie comme support, afin de questionner d'une manière inédite l'énigme du corps. En interpellant les créatures fantomatiques auxquelles Ernest Breleur donne vie, l'auteur affirme avec conviction que l'art a « le pouvoir d'inventer ce qui manque et de donner corps à ce qui manque" (Les corps énigmatiques d'Ernest Breleur, Paris, Éd. L'Harmattan, 2006, p. 74). Comment, face à cette irréductible (et fascinante) particularité de l'œuvre d'art, envisager la construction d'un discours critique à son encontre? Tel est bien, finalement, le propos central de l'essai que nous évoquons ici.

2 En effet, quelle posture adopter face à un objet, l'œuvre d'art, qui, sans cesse, se dérobe? Dans sa Théorie esthétique, Theodor $\mathrm{W}$. Adorno écrit que nous ne pouvons ignorer l'autonomie (fut-elle relative) de l'œuvre et qu'il est donc nécessaire de se confronter au «langage polémique » qui se déploie au travers de sa forme. L'œuvre 
d'art, qui n'est pas une simple chose parmi les choses, nous propose de faire l'expérience d'une logique et d'une cohérence qui lui sont propres. Par son identité bien souvent étrange, par sa configuration complexe, par sa modalité d'existence décalée, l'œuvre d'art, radicalement asociale, assume pleinement sa singularité et sa présence au monde dissonantes. Pour le théoricien de l'école de Francfort, intégrant des éléments issus du réel empirique, "elle les transpose, les décompose et les reconstruit selon sa propre loi " (Théorie esthétique, trad. de l'allemand par M. Jimenez, Paris, Klincksieck, 1974, p. 342). Réalité sui generis, ni loquace ni transparente, étrangère à un quelconque processus communicationnel, l'œuvre, par le moment de la forme, se distingue donc de l'existant. Comment, dès lors que l'on refuse de s'en tenir à un insatisfaisant et problématique silence face à elle, la critiquer ? Comment, questionne plus précisément Dominique Berthet, en rejetant tout propos dogmatique et normatif, "parler d'une œuvre avec l'œuvre elle-même » (p. 7) ? Et ce, tout en sachant, comme l'observait déjà à juste titre Gœthe, que pratiquer la critique n'est pas chose aisée !

Dans un premier temps, trois grandes figures retiennent l'attention de Dominique Berthet. L'auteur insiste d'abord sur l'importance d'un ouvrage de Lessing, Laocoon ou Des frontières de la peinture et de la poésie (1766), remettant en question la théorie dominante alors de l'ut pictura poesis. S'attachant à distinguer la spécificité picturale au regard de la création poétique, cette récusation permet à la critique d'art de disposer de nouveaux critères d'appréciation de l'œuvre peinte. Lessing insiste en fait sur la question des formes et des couleurs et sur la capacité de la peinture à rendre « dans l'instant l'impression visuelle» (p. 17). Dominique Berthet s'intéresse ensuite à la pratique critique de Diderot, qui, note-t-il, peut être considéré comme « le fondateur de la critique d'art sous sa forme moderne » (p. 21). En effet, Diderot ne décrit ni ne commente les œuvres d'art; son objectif est de «rendre visible la peinture» (p. 22). S'opposant à toute critique systématique, il privilégie une critique philosophique en quête de l'idée de l'œuvre (qui, pour lui, dépasse le faire). Pour Diderot, constate l'auteur, une peinture sans expression est par ailleurs « froide, sans imagination, sans verve» (p. 26). C'est donc l'émotion et le plaisir que suscite le tableau qui sont privilégiés. Aussi la critique, fondée sur l'opinion du critique, sur sa subjectivité et sur son engagement, doit-elle insister sur la puissance fictionnelle et « persuasive » (p. 32) de l'œuvre. Enfin, Dominique Berthet s'attarde sur celui qui, tout en s'inspirant de Diderot, développe une posture critique originale et profondément moderne, à savoir Baudelaire. En quelques pages, aussi claires qu'enthousiastes, il montre que le poète " aspire à élaborer une philosophie de l'art en partant de l'actualité artistique » (p. 40). Relisant avec rigueur les textes consacrés aux Salons ou au peintre de la vie moderne, analysant avec vigueur les arguments développés contre l'art réaliste et contre la doctrine de l'art pour l'art, Dominique Berthet rend hommage à la capacité de Baudelaire de saisir les fondements de la modernité en constituant « l'archéologie qui rend compte de notre présent » (p. 52). Au-delà, il loue la place que ce dernier accorde à l'imagination (pour Baudelaire, l'imagination est « la reine du vrai»), «le don le plus précieux [...] que l'artiste doit soigneusement préserver face à la société qui ne dissimule pas son hostilité vis-à-vis de l'artiste » (p. 48).

Dans un second temps, Dominique Berthet évoque longuement l'approche sociologique de l'art. Pour lui, Erwin Panofsky, en contextualisant l'œuvre d'art, propose une « histoire de l'esprit humain tel qu'il se manifeste à travers ses œuvres artistiques » (p. 54). En rejetant la traditionnelle opposition entre la forme et le contenu (le critique aborde l'œuvre selon trois niveaux dont l'articulation se révèle complexe : la forme, le 
sujet et le contenu), en privilégiant l'approche iconologique (qu'il distingue d'une analyse iconographique), Erwin Panofsky s'attache à saisir la signification de l'œuvre, incitant ainsi la critique d'art à s'aventurer dans un processus de «re-création » selon l'expression utilisée par Bernard Teyssèdre. S'attardant par ailleurs sur les travaux de Pierre Francastel, Dominique Berthet montre que la sociologie de l'art doit prendre en considération le fait que l'œuvre d'art renvoie à «l'imaginaire» de l'artiste et "informe sur les activités fondamentales de l'homme» (p. 62). En effet, Pierre Francastel, soulignant la nécessité de se confronter à la pensée plastique, invite le sociologue à s'engager dans une véritable enquête face à l'œuvre, envisagée, dans ses Études de sociologie de l'art, (Paris, Denoël, 1970) comme un "lieu de convergence » (et non un simple reflet du réel) au sein duquel se télescopent diverses visions de l'homme et du monde. De même, l'auteur dresse un intéressant panorama de la critique contemporaine en discutant, pour ne citer que quelques exemples, les positions défendues par Jean-François Lyotard qui insiste sur l'« imagination expérimentatrice » du commentaire, par Frank Popper qui souhaite écrire "avec l'art», par Gilbert Lascault qui préconise une critique-fiction portée par un langage poétique, par Rainer Rochlitz qui s'efforce de définir quelques critères « rationnellement justifiables » (p. 83) pour évaluer la pertinence et la réussite de l'œuvre ou encore par Guy Scarpetta, qui, alors que la puissance subversive (politique?) de l'œuvre semble s'estomper aujourd'hui, construit une esthétique du trouble. Dominique Berthet aborde également avec pertinence les relations qu'entretiennent la critique d'art et l'esthétique, en consacrant notamment un important chapitre à Walter Benjamin, pour qui la critique, inévitablement liée "à la situation historique et sociale » (p. 96) au sein de laquelle elle opère, doit avoir pour objectif de faire surgir la teneur de vérité de l'œuvre.

En conclusion, rappelant que, selon Umberto Eco, l'œuvre est ouverte, Dominique Berthet précise ce que doit être, entre passion et raison, une authentique critique d'art. Alors que, trop souvent désormais, la critique, parce que le critique se comporte comme un informateur ou un publiciste, ne semble plus vraiment remplir le rôle que l'on peut attendre d'elle, il précise, sans mépriser le plaisir esthétique, mais en en mentionnant que celui-ci «relève de l'émotion privée » (p. 107), que loin de traduire et d'épuiser l'œuvre, considérée comme une énigme, la critique, qui est « un jugement destiné à l'autre» (p. 106), se doit de l'interpréter et de la prolonger, autrement dit de produire du sens.

\section{AUTEURS}

\section{JEAN-MARC LACHAUD}

CREM, université Paul Verlaine-Metz 